\title{
Influência do Ganho de Peso Materno no Estado Nutricional do Recém-Nascido
}

\author{
Carlos Igor Mazzitelli Balsamo, ${ }^{1}$ Vinícius Vargas Dal Carobo, ${ }^{2}$ Marcia Montagner, ${ }^{2}$ \\ Camila Lehnhart Vargas, ${ }^{2}$ Franceliane Jobim Benedetti ${ }^{2}$
}

\begin{abstract}
RESUMO
Este estudo tem como objetivo analisar a influência do ganho de peso materno sobre o estado nutricional do recém-nascido (RN). Trata-se de um estudo quantitativo com delineamento transversal. A amostra foi constituída por mães e bebês nascidos em uma maternidade de risco habitual. Nas primeiras 48 horas após o parto realizaram-se: a coleta de dados do prontuário médico, do cartão da gestante e as entrevistas com a puérpera. A coleta de dados constituiu-se por meio de um instrumento de avaliação, em que estavam divididos em bloco: gestação, mãe e criança. Foram avaliados 184 mães e RNs; a média do peso pré-gestacional ficou em $60,96 \pm 11,57 \mathrm{~kg}$ e a média do ganho de peso gestacional foi de $13 \pm 5,18 \mathrm{~kg}$, com frequência elevada de inadequações. Destaca-se que as puérperas com ensino médio e das classes econômicas D-E, que apresentaram ganho de peso insuficiente ou superior ao recomendado, tiveram consecutivamente RNs classificados abaixo ou acima do peso recomendado para idade gestacional. Sendo assim, o acompanhamento do ganho de peso gestacional é fundamental, pois pode, quando associado à escolaridade e à classificação econômica, interferir no estado nutricional dos RNs.

Palavras-chave: Estado nutricional. Gravidez. Recém-nascido.
\end{abstract}

\section{INFLUENCE OF MATERNAL WEIGHT GAIN ON THE NEWBORN'S NUTRITIONAL STATUS}

\section{ABSTRACT}

This study aims to analyze the influence of maternal weight gain on the newborn's nutritional status. This is a quantitative study with a cross-sectional design. The sample consisted of mothers and babies born in a maternity at usual risk. In the first 48 hours after delivery, data were collected from the medical records, the pregnant woman's card and the interviews with the postpartum woman. Data collection was constituted by means of an evaluation instrument, in which they were divided into block: pregnancy, mother and child. A total of 184 mothers and newborns were evaluated, the mean pre-gestational weight was $60.96 \pm 11.57 \mathrm{~kg}$, and the mean gestational weight gain was $13 \pm 5.18 \mathrm{~kg}$, with a high frequency of inadequacies. It is noteworthy that the puerperal women with high school and from economic classes D-E, who presented insufficient or higher weight gain than recommended and had newborns classified below or above the recommended for gestational age. Thus, the monitoring of gestational weight gain is fundamental, as they can when associated with schooling and economic classification can interfere in the nutritional status of newborns.

Keywords: Nutritional status. Pregnancy. Newborn.

\footnotetext{
Autor correspondente. Universidade Franciscana - UFN. R. dos Andradas, 1614 - Centro, 97010-030. Santa Maria/RS, Brasil. http://lattes.cnpq.br/6775868163456909. https://orcid.org/0000-0003-4763-7802. carlosmazzitelli12@gmail.com

2 Universidade Franciscana - UFN. Santa Maria/RS, Brasil.
} 


\section{INTRODUÇÃO}

O estado nutricional materno, assim como o ganho de peso gestacional (PG), são focos atuais de estudos não apenas pela crescente prevalência dos seus distúrbios associados, mas, sobretudo, em razão de seu papel determinante sobre os desfechos gestacionais e ao longo da vida (WELLS et al., 2006; MAX, 2009; TEIXEIRA; CABRAL, 2016; SILVA et al., 2018).

O ganho ponderal no decorrer da gestação é um fator importante para o crescimento fetal, por isso recomendações para ganho de peso adequado vêm sendo propostas ao longo das últimas décadas (WELLS et al., 2006; FURLAN; CARLI; KÜMPEL, 2019). $O$ aumento de peso inadequado durante a gestação pode contribuir significativamente para a epidemia mundial de obesidade nos tempos modernos (PIRES et al., 2020). Além dos fatores fisiológicos, a obtenção de peso durante a gestação também está associada a fatores nutricionais, sociodemográficos, obstétricos e comportamentais (DREHMER et al., 2010).

A prevalência do ganho de peso excessivo durante o período gestacional vem aumentando e sendo motivo de preocupação entre os profissionais de saúde. Desta forma, ressalta-se a importância de prevenir o aumento de peso excessivo a fim de minimizar os riscos para a saúde materna e fetal (BODNAR et al., 2011). Ainda, é essencial que os profissionais da saúde, principalmente os nutricionistas, continuem promovendo e incentivando as gestantes quanto à adoção de hábitos alimentares mais saudáveis para prevenir o excesso de peso e complicações ao longo da gestação (FURLAN; CARLI; KÜMPEL, 2019).

Estudos demonstraram que as mulheres que estão com sobrepeso no início da gravidez são significativamente mais pesadas após o parto e tendem a não recuperar o peso pré-gestacional (NOGUEIRA; CARREIRO, 2013; SILVA et al., 2014). Mulheres com Índice de Massa Corporal (IMC) acima de $25 \mathrm{~kg} / \mathrm{m}^{2}$ antes de engravidar são mais propensas a apresentarem resultados adversos relacionados à gravidez, como diabetes mellitus gestacional (DMG), hipertensão induzida pela gravidez, pré-eclâmpsia, infecções puerperais, parto cirúrgico e complicações neonatais, como a hipoglicemia neonatal, quando comparadas com peso pré-gestacional abaixo do IMC $25 \mathrm{~kg} / \mathrm{m}^{2}$ (GUELINCKX et al., 2008; SILVA et al., 2014).

Neste contexto percebe-se a importância de os profissionais que atuam nas áreas de ciências da saúde e humanas ampliarem o seu pensar e seu agir de maneira gradativa, fortalecendo efetivamente as ações contempladas no Sistema Único de Saúde (SUS), o qual exige uma atuação profissional prospectiva e participativa (BRASIL, 2012).

Diante dessas questões, salienta-se a relevância de avaliar as intercorrências do ganho de peso inadequado na gestação para a saúde nutricional do $\mathrm{RN}$, pois, a partir delas, pode-se construir bases para a elaboração de estratégias que minimizem os fatores de riscos e o ganho de peso inadequado da gestante e do RN.

Proporcionar um ganho de PG adequado para a gestante e RN é fundamental, uma vez que oferecer qualidade de vida no futuro para os mesmos diminui a possibilidade do desenvolvimento de doenças crônicas como diabetes mellitus, retenção de peso pós-parto, hipertensão arterial, entre outras. Além disso, destaca-se que, apesar da relevância do tema, estudos atuais são escassos na literatura. Assim, este estudo tem como objetivo analisar a influência do ganho de peso materno sobre o estado nutricional do RN.

\section{METODOLOGIA}

Este trabalho fez parte do projeto denominado "Desenvolvimento e crescimento de lactentes: uma coorte de nascimento", o qual tem aprovação no Edital Programa Primeiros Projetos - ARD/PPP 2014 - e pelo Comitê de Ética em Pesquisa (CEP) sob o parecer número 2.118.195.

Trata-se de um estudo quantitativo com delineamento transversal. A amostra foi constituída por mães e bebês nascidos em uma maternidade pública de risco habitual em uma cidade do Centro do Estado do Rio Grande do Sul. Nas primeiras 48 horas após o parto realizaram-se: a coleta de dados do prontuário médico, do cartão da gestante e as entrevistas com a puérpera. Os critérios de inclusão contemplaram mães que tiveram o nascimento de seus bebês na maternidade pública nas últimas $48 \mathrm{~h}$ e residentes na área urbana do município. Os critérios de exclusão foram: mães que estão sob tratamento psiquiátrico, e RNs com idade gestacional inferior a 37 semanas, portadores de malformações em geral e/ou que contemplem problemas genéticos que necessitem de internação em Unidade de Tratamento Intensivo Neonatal logo após o parto, posto que a maternidade não é de alta complexidade, e os que o destino é a adoção.

A coleta de dados constituiu-se por meio de um instrumento de avaliação em que estavam divididos em blocos: gestação, mãe e criança. 
No bloco gestação avaliou-se o número de consultas pré-natal, as intercorrências na gestação, o peso pré-gestacional e o ganho de PG; no bloco mãe, realizou-se a avaliação da história reprodutiva, a idade, a escolaridade e a classificação econômica, e no bloco da criança verificou-se o índice de apgar, a idade gestacional, o peso e o comprimento de nascimento.

As intercorrências gestacionais, peso pré-gestacional, ganho de PG e o número de consultas pré-natal foram obtidos da caderneta de saúde da gestante e seguiu-se as recomendações do Ministério da Saúde (MS) e Instituto de Medicina (IOM) (BRASIL, 2011; IOM, 2009). A classificação econômica foi analisada de acordo com o Critério de Classificação Econômica do Brasil da Associação Brasileira de Empresas de Pesquisa (Abep), que distribui as classes em A, B1, B2, C1, C2 e D-E. A classificação quanto à escolaridade também foi agrupada conforme a recomendação descrita no instrumento (ABEP, 2015).

$O$ índice de apgar, o peso, a estatura de nascimento e a idade gestacional foram coletados em prontuário médico conforme ultrassom ou data da última menstruação (BRASIL, 2011). Para obtenção do z-escore do peso e estatura de nascimento para idade gestacional, utilizaram-se as curvas e a calculadora Intergrowth-21st, consideradas padrão internacional de crescimento, destinadas para o monitoramento do crescimento fetal e de RN, (INTERGROWTH-21st, 2020). Salienta-se que estas curvas estão inseridas na nova Caderneta da Criança (BRASIL, 2020). A classificação dos z-escores seguiu as recomendações do Intergrowth-21st, que considera adequados os RNs que estão entre os z-escores +1 e -1 (INTERGROWTH-21st, 2020).

As informações coletadas foram armazenadas no Microsoft Excel e passaram por tratamento estatístico no software Statistical Package for the Social Sciences (SPSS), versão 23.0. Para verificar a normalidade da amostra aplicou-se o teste de aderência Kolmogorov-Smirnov. Para descrever as variáveis qualitativas nominais e ordinais utilizaram-se as frequências absolutas e relativas, e para as quantitativas contínuas a média e o desvio-padrão.

A diferença entre o ganho de peso materno e peso pré-gestacional em relação à classificação do peso para a idade gestacional de RNs foi analisada por meio do teste Anova, post-hoc Tukey. Já a associação entre as variáveis econômicas, demográficas e o pré-natal, foi pelo teste de qui-quadrado de Pearson. A correlação entre ganho de peso da mãe com peso, estatura para idade e z-score do RN, foi obtida pelo teste de correlação de Pearson. Considerou-se o nível de significância de $5 \%(p<0,5)$.

\section{RESULTADOS}

Neste estudo foram avaliados 184 mães e RNs. As mães apresentaram média de idade de $23,73 \pm 5,74$ anos e a classe econômica " $C$ " foi a mais prevalente. Quanto à escolaridade, a maioria das mães completou o Ensino Fundamental e Médio. Do total de mães avaliadas, 178 realizaram as consultas pré-natal, e, destas, $132(79,0 \%)$ realizaram mais de 6 consultas.

Em relação ao número de gestações anteriores, a mediana foi de $1(0 / 2)$ gestação. Também se observou que a média do peso pré-gestacional materno foi de $60,96 \pm 11,57 \mathrm{~kg}$, e a média do ganho de PG foi de $13,0 \pm 5,18 \mathrm{~kg}$, posto que $57(32,9 \%)$ apresentaram ganho de peso insuficiente, $82(47,4 \%)$ adequado e $34(19,7 \%)$ superior ao recomendado. No decorrer da gestação, 50 mães tiveram algum tipo de intercorrência, 17 (34,0\%) relataram infecção urinária, 5 (10,0\%) anemia e $4(8,0 \%)$ cefaleia.

Os RNs apresentaram, em média, $39,0 \pm 1,10$ semanas de idade gestacional, e os índices de apgar no $5^{\circ}$ minuto foram superiores ao 1 으 (Tabela 1 ), sendo os valores mínimos de 4 e 8 pontos para o $1^{\circ}$ e $5^{\circ} \mathrm{mi}-$ nutos, consecutivamente, e os valores máximos foram de 10 pontos nos dois tempos.

A média do peso e estatura foi $3317,0 \pm 439,63$ gramas e $48,0 \pm 1,96$ centímetros. A mediana do peso e estatura para idade gestacional estão apresentadas na Tabela 1. Observa-se que $145(81,0 \%)$ dos RNs encontram-se adequados para a idade gestacional, 16 $(8,9 \%)$ abaixo do z-escore -1 e $18(10,1 \%)$ acima do z-escore +1 .

Tabela 1 - Descrição das variáveis de saúde das puérperas e dos recém-nascidos

\begin{tabular}{lc}
\hline Fatores & Média/Mediana \\
\hline Gestação/Puérpera & \\
Peso Mãe $(\mathrm{kg})$ & $60,96 \pm 11,57^{*}$ \\
Ganho de Peso $(\mathrm{kg})$ & $13,41 \pm 5,18^{*}$ \\
Gestações Anteriores & $1(0 / 2)^{* *}$ \\
Número de Partos & $2(1 / 2)^{* *}$ \\
Número de abortamentos & $1,04 \pm 0,21^{*}$ \\
Número de Consultas Pré-natal & $9,17 \pm 3,29^{*}$
\end{tabular}


Recém-nascido

\begin{tabular}{lc} 
Idade Gestacional (semanas) & $39,58 \pm 1,10^{*}$ \\
Apgar - 10 minuto & $9,31 \pm 0,85^{*}$ \\
Apgar - 5o minuto & $9,91 \pm 0,31^{*}$ \\
Peso (g) & $3317 \pm 439,63^{*}$ \\
Estatura (cm) & $48,11 \pm 1,95^{*}$ \\
P/IG (z-escore) & $0,08(-$ \\
E/IG (z-escore) & $0,49 / 0,60)^{* *}$ \\
& $-0,79(-1,28 /-$ \\
\hline
\end{tabular}

P, peso; E, estatura; IG, idade gestacional. Valores acrescentados em *média \pm desvio padrão ou **mediana (percentis 25/75).

Fonte: Elaborada pelos autores.

Na Tabela 2 mostra-se a análise da associação entre as variáveis econômicas, demográficas e as consultas de pré-natal, associada ao ganho de peso na gestação e o peso para a idade gestacional de nascimento dos RNs. Observou-se associação estatisticamente significativa entre as puérperas com ensino médio e das classes econômicas $D-E$, ou seja, este grupo de mães apresentou ganho de peso insuficiente ou superior ao recomendado e tiveram consecutivamente RNs classificados abaixo ou acima do peso recomendado para idade gestacional.

Na Tabela 3 observa-se a média do peso pré-gestacional e do ganho de peso materno com a classificação do peso para a idade gestacional dos RNs. Destaca-se que, apesar de não haver diferença estatisticamente significativa, observou-se que as puérperas que obtiveram maior média de ganho de PG foram as que tiveram os RNs acima do ganho de peso recomendado para a idade gestacional.

Tabela 2 - Análise da frequência da classificação dos recém-nascidos conforme o ganho de peso gestacional e características econômicas, demográficas e pré-natal de puérperas de risco habitual

\begin{tabular}{|c|c|c|c|c|c|}
\hline & \multirow{2}{*}{$\begin{array}{c}\text { Ganho de Peso } \\
\text { gestacional }\end{array}$} & \multicolumn{3}{|c|}{ Recém-nascido (P/IG) } & \multirow[b]{2}{*}{$\mathbf{P}$} \\
\hline & & $\begin{array}{l}\text { Abaixo } \\
(n=16)\end{array}$ & $\begin{array}{l}\text { Adequado } \\
(n=145)\end{array}$ & $\begin{array}{l}\text { Acima } \\
(n=18)\end{array}$ & \\
\hline \multicolumn{6}{|l|}{ Escolaridade } \\
\hline \multirow[t]{3}{*}{ Fundamental } & Insuficiente & $1(3,4)$ & $4(13,8)$ & - & 0,313 \\
\hline & Adequado & - & $12(41,4)$ & $3(10,0)$ & \\
\hline & Superior & $1(3,4)$ & $5(17,2)$ & $3(10,3)$ & \\
\hline \multirow[t]{3}{*}{ Médio } & Insuficiente & $5(4,0)$ & $41(32,5)$ & $1(0,8)$ & $0,015^{*}$ \\
\hline & Adequado & $3(2,4)$ & $51(40,5)$ & $3(2,4)$ & \\
\hline & Superior & $3(2,4)$ & $14(11,1)$ & $5(4,0)$ & \\
\hline \multirow[t]{3}{*}{ Superior } & Insuficiente & - & - & - & 0,102 \\
\hline & Adequado & $1(9,1)$ & $7(63,6)$ & - & \\
\hline & Superior & - & $1(9,1)$ & $1(9,1)$ & \\
\hline \multicolumn{6}{|c|}{ Classificação econômica } \\
\hline \multirow[t]{3}{*}{$A-B$} & Insuficiente & $3(5,5)$ & $12(41,8)$ & $1(1,8)$ & 0,062 \\
\hline & Adequado & $3(5,5)$ & $23(41,8)$ & $1(1,8)$ & \\
\hline & Superior & $2(3,6)$ & $6(10,9)$ & $4(7,3)$ & \\
\hline \multirow[t]{3}{*}{ C } & Insuficiente & $2(2,4)$ & $22(26,5)$ & $1(1,2)$ & 0,428 \\
\hline & Adequado & $1(1,2)$ & $40(48,2)$ & $4(4,8)$ & \\
\hline & Superior & $2(2,4)$ & $10(12)$ & $1(1,2)$ & \\
\hline \multirow[t]{3}{*}{$D-E$} & Insuficiente & $1(3,4)$ & $12(41,4)$ & - & $0,020^{*}$ \\
\hline & Adequado & - & $8(27,6)$ & $1(3,4)$ & \\
\hline & Superior & - & $3(10,3)$ & $4(13,8)$ & \\
\hline \multicolumn{6}{|l|}{ Pré-natal } \\
\hline \multirow[t]{3}{*}{$>6$ consultas } & Insuficiente & $1(3,4)$ & $7(24,1)$ & $1(3,4)$ & 0,145 \\
\hline & Adequado & - & $12(41,4)$ & $2(6,9)$ & \\
\hline & Superior & $1(3,4)$ & $2(6,9)$ & $3(10,3)$ & \\
\hline \multirow[t]{3}{*}{$<6$ consultas } & Insuficiente & $5(4,0)$ & $33(26,6)$ & $1(0,8)$ & 0,167 \\
\hline & Adequado & $4(3,2)$ & $54(43,5)$ & $4(3,2)$ & \\
\hline & Superior & $3(2,4)$ & $16(12,9)$ & $4(3,2)$ & \\
\hline
\end{tabular}

P, peso; IG, idade gestacional. Valores apresentados em $n$ (\%). Teste de qui-quadrado de Pearson, * $p<0,05$. 
Tabela 3 - Média do peso pré-gestacional e ganho de peso materno na gestação em relação à classificação do peso para idade gestacional de recém-nascidos em hospital de risco habitual

\begin{tabular}{|c|c|c|c|c|c|c|c|}
\hline \multirow{3}{*}{$\begin{array}{l}\text { Variáveis } \\
\text { maternas }\end{array}$} & \multicolumn{4}{|c|}{ Recém-nascido (P/IG) } & & & \multirow{3}{*}{$p^{*}$} \\
\hline & \multirow{2}{*}{$\begin{array}{l}\text { Abaixo } \\
(\mathrm{n}=16)\end{array}$} & & \multicolumn{2}{|l|}{$\begin{array}{c}\text { Adequado } \\
(n=145)\end{array}$} & \multicolumn{2}{|l|}{$\begin{array}{l}\text { Acima } \\
(\mathrm{n}=18)\end{array}$} & \\
\hline & & DP & Média & DP & Média & DP & \\
\hline $\begin{array}{l}\text { Peso } \\
\text { pré-gestacional }\end{array}$ & 59,62 & 12,99 & 60,80 & 11,22 & 66,63 & 12,14 & 0,123 \\
\hline Ganho de peso gestacional & 12,10 & 4,57 & 12,82 & 5,07 & 15,70 & 6,38 & 0,078 \\
\hline
\end{tabular}

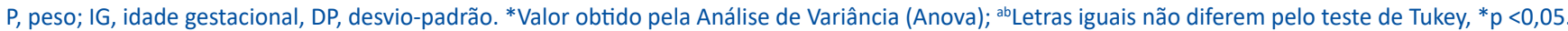
Fonte: Elaborada pelos autores.

A correlação entre o ganho de peso materno e os indicadores antropométricos (P/IG e E/IG) apresentados em z-escore estão apresentados na Figura 1. Observa-se que as mesmas foram positivas, porém fracas.

\section{DISCUSSÃO}

O acompanhamento do ganho de peso na gestação é de suma importância, pois a gestante necessita de ajuda para compreender suas novas necessi- dades. Sendo assim, o pré-natal é o momento ideal para esta avaliação. Neste sentido, este estudo avaliou puérperas internadas em uma maternidade de risco habitual e observou que a maior parte delas são de classes econômicas mais baixas, completaram o Ensino Fundamental e Médio e realizaram mais de seis consultas pré-natal, no entanto cerca de metade das gestantes apresentou inadequação de ganho de peso.

Figura 1 - Correlação entre ganho de peso da mãe com peso, estatura para idade e z-score do recém-nascido
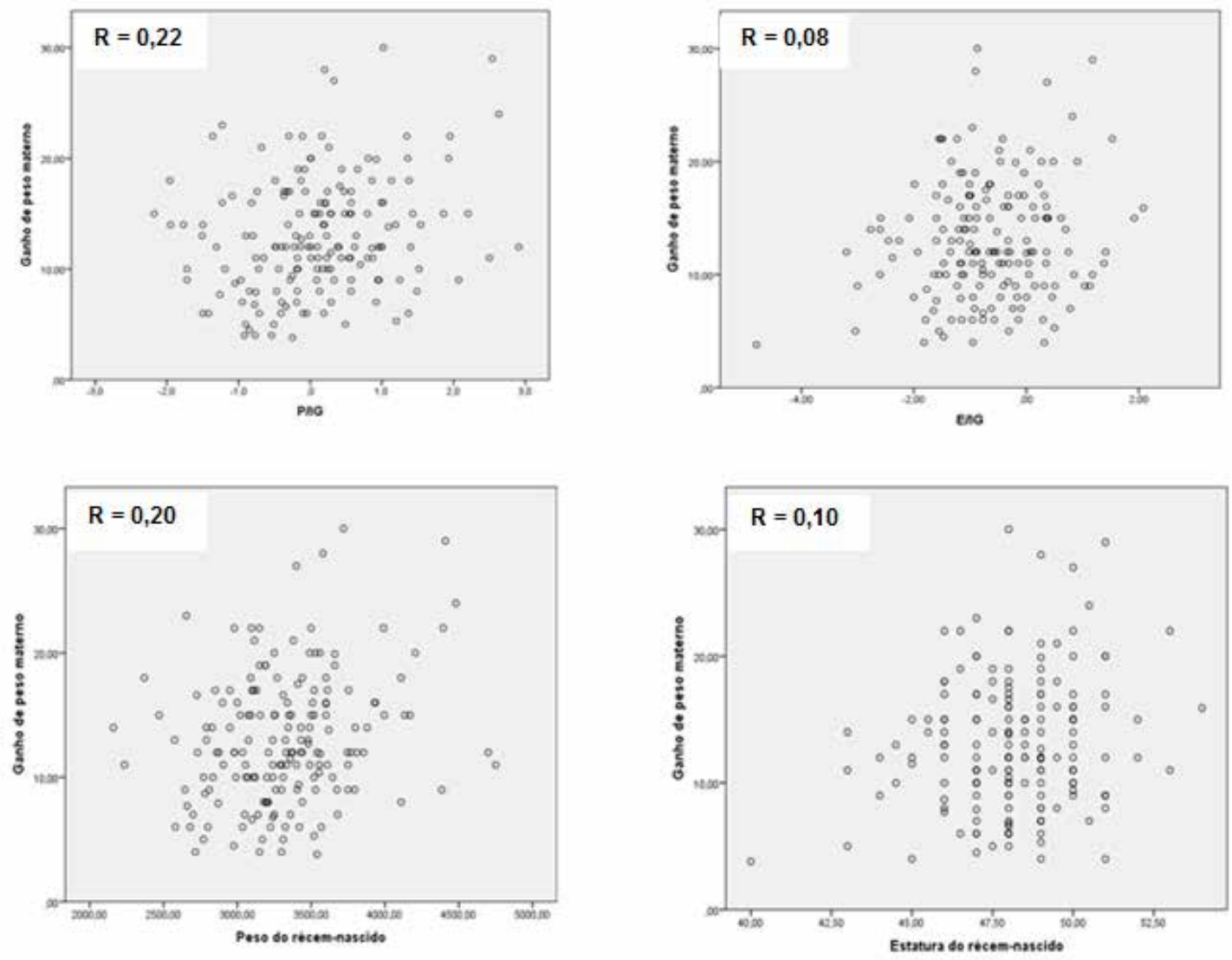

Fonte: Elaborada pelos autores. 
A maioria dos RNs mostrou bom estado geral e adequado peso para a idade gestacional, posto que 145 (81\%) deles encontraram-se com peso adequados para a idade gestacional, percentual superior ao observado no estudo de Santos et al. (2015), que avaliaram 24 puérperas e seus filhos. Dos neonatos, 79,2\% foram classificados como adequados para a idade gestacional (AIG). Em estudo realizado por Pereira e Wichmann (2016), no município de Candelária (RS), em uma amostra composta por 169 puérperas atendidas em uma Unidade Básica de Saúde (UBS), 62,7\% das crianças tiveram peso adequado, sendo inferior ao percentual do presente estudo. Já em estudo retrospectivo, que incluiu 2.849 RNs com peso ao nascer de $3210 \pm 483 \mathrm{~g}$, idade gestacional de $38,8 \pm 1,4$ semanas, observou-se incidência de $8,7 \%$ de RNs pequenos para a idade gestacional; também utilizou a curva Intergrowth-21st (BARRETO et al., 2020), com resultado similar ao presente estudo, o qual obteve $8,9 \%$ de RNs abaixo do recomendado para a idade gestacional.

As curvas Intergrowth-21st são consideradas os padrões de crescimento fetal. Elas vão além dos objetivos limitados de referências passadas. $O$ estudo de Papageorghiou et al. (2018), confirmou o princípio biológico fundamental de que a variação do crescimento humano em diferentes populações depende, principalmente, de fatores ambientais, nutricionais e socioeconômicos.

$O$ ganho de $P G$ é provocado não só pelo crescimento do feto, mas também pelo aumento do volume de sangue circulante, ganho de gordura, retenção de água, aumento da massa muscular do útero, entre outros motivos. $\mathrm{O}$ aumento de peso pode trazer alguns riscos nutricionais tanto para a puérpera quanto para o RN. Com isso, ressalta-se a importância do ganho de PG adequado e o acompanhamento pré-natal (BRASIL, 2012). No estudo realizado por Fonseca et al. (2014) a média do ganho de peso na gestação foi de $13,20 \pm 5,51 \mathrm{~kg}$; no estudo atual a média do ganho de PG foi $13,41 \pm 5,18 \mathrm{~kg}$. Dos RNs, no entanto, $32,9 \%$ apresentaram ganho de peso insuficiente, $19,7 \%$ superior ao recomendado, percentuais superiores ao observado por Santana et al. (2020), que, ao avaliarem 185 mulheres ao longo da gestação, constataram que $33,5 \%$ apresentaram ganho ponderal inadequado (superior ou inferior).

Conforme o MS, a realização do pré-natal representa papel fundamental na prevenção e/ou detecção precoce de doenças tanto maternas quanto fetais, permitindo um desenvolvimento saudável do bebê e reduzindo os riscos da gestante (BRASIL, 2012). Em estudo também realizado no Estado do RS, constatou-se que $65,9 \%$ das gestantes avaliadas, realizaram mais de seis consultas no pré-natal, sendo o percentual inferior ao do presente estudo, que teve $79 \%$ das gestantes com mais de seis consultas, conforme recomendado pelo MS (DREHMER et al., 2010).

O pré-natal é fundamental, considerando-se que algumas intercorrências na gravidez são esperadas em razão das alterações nas funções circulatórias, renais, neurológicas e metabólicas, pois ocorre mudanças no equilíbrio fisiológico do organismo da muIher. No estudo de Fonseca et al. (2014), os autores observaram algumas intercorrências durante a gestação: $177(54,6 \%)$ tiveram infecção do trato urinário e $44(13,6 \%)$ anemia. Comparado com o atual estudo, 17 (34\%) indicaram infecção urinária e 5 (10\%) relataram anemia. Duarte et al. (2008) e Darzé, Barroso e Lordelo (2011) descreveram que as alterações anatômicas e fisiológicas que ocorrem no sistema urinário durante a gravidez facilitam ainda mais o desenvolvimento de infecções urinárias sintomáticas em muIheres que apresentaram bacteriúria anteriormente. Essas condições podem levar ao agravamento, tanto para o prognóstico materno quanto para o prognóstico perinatal.

Além do pré-natal, os fatores demográficos e econômicos são aspectos importantes para uma maior adesão do acompanhamento pré-natal, o que, consequentemente, proporciona um desfecho nutricional positivo para puérpera e RN. Neste sentido, no presente estudo destaca-se que $134(73,6 \%)$ das mães tinham Ensino Fundamental e Ensino Médio. Em estudo realizado por Moreira et al. (2017), 131 (48,9\%) das mães tinham escolaridade superior a 8 anos e 137 $(51,1 \%)$ tinham escolaridade inferior ou igual a 8 anos. Quanto à classificação econômica, em estudo realizado por Dell'Osbel, Cremonese e Gregoletto (2019), $68,1 \%$ das puérperas encontram-se nas classes C-E, e $25,5 \%$ na classe E. Já os resultados e método de análise de Furlan, Carli e Kümpel (2019) foram semeIhantes aos do presente estudo, pois mostraram que $60,9 \%$ das gestantes encontravam-se na classe $C$.

A relação do peso materno com o estado nutricional do RN e os fatores associados ainda são controversos. No presente estudo a correlação do peso pré-gestacional e PG foi positiva, porém fraca; entretanto ao associar o peso para idade gestacional dos RNs com as variáveis econômicas e demográficas e pré-natal, observou-se que mães que apresentam Ensino Médio ou estavam na classificação econômica D-E, com ganho de peso insuficiente na gestação, tiveram 
RNs com P/IG abaixo do recomendado, e aquelas que tiveram ganho de peso superior, tiveram RNs acima do recomendado.

O estudo realizado por Santos et al. (2015) concluiu, após ajustes da regressão logística, que o peso ao nascer foi associado ao estado nutricional pré-gestacional $(O R=5,457 ; p=0,012)$, início do pré-natal $(\mathrm{OR}=1,509 ; \mathrm{p}=0,015)$ e densidade de cálcio dietético $(\mathrm{OR}=2,672 ; \mathrm{p}=0,048)$. Já o estudo de Dell'Osbel, Cremonese e Gregoletto (2019) identificou associação significativa entre o ganho de PG insuficiente com a baixa escolaridade e ao menor comprimento ao nascer. Mais recentemente, o ganho de peso inadequado no período gestacional foi considerado fator de risco para o peso ao nascer $(O R=2,6$; IC95\%= 1,5-3,5), mesmo quando ajustado pelas variáveis nutricionais e intercorrências gestacionais (SANTANA et al., 2020).

Os resultados deste estudo, em colaboração com os supracitados, sugerem que o ganho de PG pode influenciar o peso do RN, e sugerem, na sua maioria, que as intervenções nutricionais sejam realizadas durante o pré-natal com a finalidade de acompanhar o peso da gestante, promovendo um ganho ponderal saudável, além de monitorar outros fatores de risco que podem contribuir para a redução de inadequações do peso de nascimento.

\section{CONCLUSÃO}

O ganho de PG é um importante indicador de saúde materno; no entanto, quando se observa altas frequências de inadequações, como as encontradas no presente estudo, associadas à escolaridade e classificação econômica, podem influenciar no estado nutricional dos RNs. Neste sentido, salienta-se a importância do acompanhamento de peso durante as consultas de pré-natal, além de uma exploração maior do tema, pois o estado nutricional adequado, tanto da mãe quanto do RN, pode proporcionar uma melhor qualidade de vida não só momentânea, mas futura.

\section{REFERÊNCIAS}

ABEP. Associação Brasileira de Empresas de Pesquisa. Critério de Classificação Econômica Brasil. 2015. Disponível em: http://www.abep.org/. 3 set. 2020.

BARRETO, C. M. et al. Incidência de recém-nascidos pequenos para a idade gestacional segundo curva de Fenton e Intergrowth-21st em uma maternidade de nível secundário. Rev. Paul. Pediatr., São Paulo, v. 39, p. 1-9, e2019245, 2020.

BODNAR, L. M. et al. Should gestational weight gain recommendations be tailored by maternal characteristics? Am J Epidemiol., v. 174, n. 2, p. 136-46, 2011.
BRASIL. Ministério da Saúde. Secretaria de Atenção à Saúde. Departamento de Ações Programáticas e Estratégicas. Atenção à saúde do $R N$ : guia para os profissionais de saúde. Ministério da Saúde, Secretaria de Atenção à Saúde, Departamento de Ações Programáticas e Estratégicas. Brasília: Ministério da Saúde, 2011.

BRASIL. Ministério da Saúde. Secretaria de Atenção à Saúde. Departamento de Atenção Básica. Atenção ao pré-natal de baixo risco. Brasília: Editora do Ministério da Saúde, 2012.

BRASIL. Ministério da Saúde. Secretaria de Atenção Primária à Saúde. Caderneta da Criança. Passaporte da Cidadania. 2. ed. 2020.

DARZÉ, O. I. S. P.; BARROSO, U.; LORDELO, M. Preditores clínicos de bacteriúria assintomática na gestação. Rev. Bras. Ginec. Obst., v. 33, n. 8, p. 196-200, 2011.

DELL'OSBEL, R.; CREMONESE, C.; GREGOLETTO, M. Ganho de peso gestacional e fatores associados em gestantes e recém-nascidos. Revista Contexto \& Saúde, v. 19, n. 37, p. 20-29, 2019.

DREHMER, M. et al. Socioeconomic, demographic and nutritional factors associated with maternal weight gain in general pratices in Southern Brazil. Cadernos de Saúde Pública, v. 26, n. 5, p. 1.024-1.034, 2010.

DUARTE, G. et al. Urinary tract infection in pregnancy. Rev. Bras. de Ginec. e Obst., v. 30, n. 2, p. 93-100, 2008.

FONSECA, M. R. C. C. et al. Ganho de peso gestacional e peso ao nascer do concepto: estudo transversal na região de Jundiaí, São Paulo, Brasil. Ciência \& Saúde Coletiva, v. 19, n. 5, p. 1.401-1.407, 2014.

FURLAN, C.; DE CARLI, G.; KÜMPEL D. S. Excesso de peso e consumo alimentar de gestantes atendidas em unidades básicas de saúde. Saúde, Santa Maria, v. 45, n. 2, p. 12, 2019.

GUELINCKX, l. et al. Maternal obesity: Pregnancy complications, gestational weight gain and nutrition. Obesity Reviews, v. 9, p. 140-150, 2008.

INTERGROWTH-21st. The International Fetal and Newborn Growth Consortium for the $21^{\text {st }}$ Century. University of Oxford, 2020. Disponivel em: http://http://intergrowth21. ndog.ox.ac.uk/. Acesso em: 24 mar. 2020.

IOM. Institute of Medicine. Nutrition during pregnancy. Washington DC: National Academies Press, 2009.

MAX, J. R. Risks and management of obesity in pregnancy: current controversies. Curr. Opin. Obstet. Gynecol., v. 21, n. 2, p. 117-23, 2009.

MOREIRA, M. É. F. H. et al. Determinantes socioeconômicos e gestacionais do peso ao nascer de crianças nascidas a termo. Medicina (Brazil), v. 50, n. 2, p. 83-90, 2017.

NOGUEIRA, A. I.; CARREIRO, M. N. Obesidade e gravidez. Rev. Med. Minas Gerais, v. 23, n. 1, p. 86-95, 2013.

PAPAGEORGHIOU, A. T. The Intergrowth-21st fetal growth standards: toward the global integration of pregnancy and pediatric care. American Journal of Obstetrics and Gynecology, v. 218, n. 2, p. 630-640, Feb. 2018. 
PEREIRA, V. R.; WICHMANN, F. M. A. Estado nutricional materno e peso ao nascer do bebê no município de Candelária - RS. Cinergis, v. 17, n. 1, p. 368-372, 2016.

PIRES C. C. et al. Evolução do excesso de peso em gestantes usuárias da Atenção Primária à Saúde do município de Macaé-RJ entre 2010-2018. Demetra, v. 15, p. 1-11, 2020.

SANTANA, J. M. et al. Associação entre ganho ponderal na gestação e peso ao nascer: Coorte Nisami. Rev. Bras. Saúde Mater. Infant., Recife, v. 20, n. 2, p. 421-430, 2020.

SANTOS, M. T. M. et al. Fatores relacionados ao peso ao nascer: influência de dados gestacionais. Revista Médica de Minas Gerais, v. 25, n. 2, p. 192-198, 2015.

SILVA, J. C. et al. Obesidade durante a gravidez: resultados adversos da gestação e do parto. Rev. Bras. Ginec. Obst, v. 36, n. 11, p. 509-513, 2014.

SILVA M. G. da. et al. Estado nutricional e hábitos alimentares de gestantes atendidas na atenção primária de saúde. Revista Brasileira de Ciências da Saúde, v. 24, n. 4, p. 349356, 2018.

TEIXEIRA C. S. S.; CABRAL, A. C. V. Avaliação nutricional de gestantes sob acompanhamento em serviços de pré-natal distintos: a região metropolitana e o ambiente rural. Rev. Bras. Ginec. Obst., v. 38, p. 27-34, 2016.

WELLS, C. S. et al. Factors influencing inadequate and excessive weight gain in pregnancy: Colorado, 2000-2002. Matern Child Health J., v. 10, n. 1, p. 55-62, 2006. 